\title{
Treating instabilities in a hyperbolic formulation of Einstein's equations
}

\author{
Mark A. Scheel, ${ }^{1}$ Thomas W. Baumgarte, ${ }^{2}$ Gregory B. Cook, ${ }^{1}$ Stuart L. Shapiro, ${ }^{2,3}$ and Saul A. Teukolsky ${ }^{1,4}$ \\ ${ }^{1}$ Center for Radiophysics and Space Research, Cornell University, Ithaca, New York 14853 \\ ${ }^{2}$ Department of Physics, University of Illinois at Urbana-Champaign, Urbana, Illinois 61801 \\ ${ }^{3}$ Department of Astronomy and NCSA, University of Illinois at Urbana-Champaign, Urbana, Illinois 61801 \\ ${ }^{4}$ Departments of Physics and Astronomy, Cornell University, Ithaca, New York 14853
}

(Received 15 September 1997; published 27 July 1998)

\begin{abstract}
We have recently constructed a numerical code that evolves a spherically symmetric spacetime using a hyperbolic formulation of Einstein's equations. For the case of a Schwarzschild black hole, this code works well at early times, but quickly becomes inaccurate on a time scale of (10-100) $M$, where $M$ is the mass of the hole. We present an analytic method that facilitates the detection of instabilities. Using this method, we identify a term in the evolution equations that leads to a rapidly growing mode in the solution. After eliminating this term from the evolution equations by means of algebraic constraints, we can achieve free evolution for times exceeding $10000 \mathrm{M}$. We discuss the implications for three-dimensional simulations.

[S0556-2821(98)00318-X]
\end{abstract}

PACS number(s): 04.25.Dm, 02.60.Cb, 02.70.-c, 04.20.Ex

\section{INTRODUCTION}

When solving Einstein's equations as an initial value problem, one considers spacetime as a foliation of spacelike hypersurfaces, or "slices." Einstein's equations then separate into two types: constraint equations, which relate the dynamical variables on each particular slice, and evolution equations, which describe how these variables propagate from one slice to the next. The constraints are analogous to the divergence equations in Maxwell's theory, and the evolution equations are analogous to the curl equations.

As in Maxwell's theory, the evolution equations admit solutions that violate the constraints. However, if the constraints are satisfied on the initial slice and on all spatial boundaries, then the evolution equations guarantee that the constraints are satisfied elsewhere. This permits numerical solution schemes in which only the evolution equations are explicitly solved at each time step.

Such "free evolution" schemes are desirable for several reasons. First, the constraints are typically nonlinear elliptic equations, which are difficult and costly to solve on a computer, especially in the general case of three spatial dimensions. Second, a free evolution scheme allows one to track numerical errors by monitoring the constraints at each time step.

For numerical evolution of black holes, an additional advantage of a free evolution scheme is that one can, in principle, excise a black hole from the spacetime and evolve only the exterior region, and one can do so without imposing explicit boundary conditions on the horizon. This is the basis for so-called "apparent horizon boundary condition" methods, which are thought to be crucial for long-term numerical evolution of black hole spacetimes [1-9]. However, excising a black hole from a spacetime is known to be mathematically well-defined only if the evolution equations are hyperbolic and if the characteristic curves of the hyperbolic system are "physical," that is, if they lie within the local light cone. In this case, the structure of the equations guarantees that no information, including gauge information, can emerge from the hole. For non-hyperbolic representations of general relativity such as the usual Arnowitt-Deser-Misner (ADM) [10] formulation, the evolution equations are of no mathematical type for which well-posedness has been proven, and so the suitability of these formulations for black hole excision must be determined empirically on a case-by-case basis. It is in part for this reason that much attention has been recently focused on hyperbolic representations of Einstein's equations [11-19].

A key stumbling block in numerical work, particularly in finite-difference solutions of initial value problems, is the tendency for numerical computations to become unstable. Instabilities have many origins, and the cause of any particular instability found in a numerical code is often difficult to deduce. Furthermore, if the desired analytic solution is unknown, it can be difficult to distinguish between an instability and a case in which the analytic solution simply grows without bound. Examples of the latter include systems that evolve to physical singularities (e.g., Oppenheimer-Snyder collapse evolved using geodesic slicing) and those that evolve toward coordinate singularities (e.g., a Schwarzschild black hole evolved with maximal time slicing, and several harmonic-slicing examples that become singular for certain choices of the initial lapse function [20,21]). When diagnosing instabilities in numerical simulations, it is therefore preferable to study instances in which the analytic solution is known and well-behaved.

We distinguish between two types of instabilities: a type in which the numerical finite-difference equations admit rapidly growing solutions that do not satisfy the underlying continuum differential equations and a type in which the continuum equations themselves admit growing modes that are absent in the desired solution but are excited by numerical perturbations. An example of the former type, which we will call a numerical instability because it depends on the numerical finite-difference equations, is the well-known Courant instability that can arise in explicit finite-difference solutions of hyperbolic partial differential equations (PDEs). The highfrequency modes that characterize a Courant instability do 
not satisfy the underlying differential equations.

The latter type, which we will call a "continuum" instability because the unstable mode satisfies the continuum differential equations, commonly occurs in systems of equations that admit both well-behaved and growing solutions. Although one might be interested in the well-behaved solution, the growing mode eventually dominates if it at any time acquires a nonzero amplitude via numerical errors. A simple example is the equation $\ddot{y}=y / 9$ with initial conditions $y=1, \dot{y}=-1 / 3$. For these initial conditions the unique analytic solution is $y=e^{-t / 3}$, but a naive numerical integration of this problem is unstable as it proceeds forward in time because numerical perturbations excite the growing solution $y=e^{t / 3}$.

For numerical solutions of Einstein's equations, a continuum instability may be due to a gauge mode excited by inaccuracies in numerically determined coordinate conditions. Or in the case of a free evolution scheme, it may be caused by a rapidly growing mode that satisfies the evolution equations but violates the constraints. This latter case is possible despite the fact that the evolution equations preserve the constraints, because in numerical computations neither the evolution equations nor the constraints are exactly satisfied. Constraint-violating modes have been discussed in the literature [22-24] but their importance for numerical free evolution schemes remains controversial.

Eliminating a continuum instability often requires a different approach than removing a numerical one, because these two types of instability stem from quite different sources. To remove a numerical instability, one must change the numerical algorithm (or details of the algorithm such as the size of the time step) that is used to solve the equations, so that this algorithm no longer introduces growing modes. To remove a continuum instability, one must either remove the numerical perturbations that excite the undesired solution of the continuum equations, change the numerical scheme in order to damp out this solution, or modify the continuum equations themselves (possibly including the choice of gauge) so that no growing solution is present.

In this paper we examine instabilities in a numerical free evolution code that solves a spherically symmetric blackhole spacetime. Our code, which has been described in detail elsewhere [7], is based on a hyperbolic formulation of general relativity [the "Einstein-Ricci" (ER) formulation] originally proposed by Choquet-Bruhat and York $[12,13]$. For short integration times our code performs well, but we show in Sec. III that for the case of a Schwarzschild black hole it becomes unstable and terminates on a time scale of (10-100) $M$, where $M$ is the mass of the hole. This occurs even in a gauge in which the analytic solution is regular at the horizon and time-independent. The rate at which our errors grow is independent of the numerical time discretization $\Delta t$ and the spatial discretization $\Delta r$, suggesting that the growth is due to a continuum instability rather than a numerical one.

In Sec. IV we present a method of analyzing the evolution equations that facilitates the detection of continuum instabilities. In the simplest application of this method we consider each ER evolution equation separately. For each equation, we examine the free evolution of the ER variable governed by that equation, treating all other ER variables as fixed and given by the Schwarzschild solution. We ask whether perturbations of the evolved ER variable about its Schwarzschild value grow rapidly with time. We find that most of the ER equations, when treated individually in this manner, are stable, but that one of the ER equations is sensitive to a continuum instability. A single term on the right-hand side of the unstable equation is responsible for the growing mode.

In Sec. V we construct a modified set of evolution equations that no longer contain this troublesome term. This is done primarily by using algebraic constraints to rewrite the right-hand side of one equation. We find that numerical free evolution of the modified set of equations remains accurate for times in excess of $10000 \mathrm{M}$. This substantial improvement indicates that the rapidly growing mode found by our analysis in Sec. IV is the dominant instability afflicting free evolution of the unmodified ER equations. In Sec. VI we discuss our method of stability analysis and apply it to the three-dimensional Einstein-Ricci equations, as well as to the Einstein-Bianchi [19] and ADM systems. We discuss the implications for three-dimensional free evolution schemes.

\section{EQUATIONS}

\section{A. ER formalism}

Here we summarize the fundamental variables and equations used in the ER representation of general relativity. For details of the ER formulation and a derivation of the equations, see $[12,13]$.

We write the metric in the usual $3+1$ form

$$
d s^{2}=-N^{2} d t^{2}+g_{i j}\left(d x^{i}+\beta^{i} d t\right)\left(d x^{j}+\beta^{j} d t\right),
$$

where $N$ is the lapse function, $\beta^{i}$ is the shift vector, and $g_{i j}$ is the three-metric on a spatial hypersurface of constant $t$.

Define the variables

$$
\begin{aligned}
K_{i j} & \equiv-\frac{1}{2} N^{-1} \hat{\partial}_{0} g_{i j}, \\
L_{i j} & \equiv N^{-1} \hat{\partial}_{0} K_{i j}, \\
M_{k i j} & \equiv D_{k} K_{i j}, \\
a_{i} & \equiv D_{i}(\ln N), \\
a_{0 i} & \equiv N^{-1} \hat{\partial}_{0} a_{i}, \\
a_{i j} & \equiv D_{j} a_{i} .
\end{aligned}
$$

Here $D$ is the three-dimensional covariant derivative compatible with the three-metric $g_{i j}$, the time derivative operator is

$$
\hat{\partial}_{0} \equiv \frac{\partial}{\partial t}-£_{\beta},
$$

and $£$ denotes a Lie derivative. The quantity $K_{i j}$ is the usual extrinsic curvature. 
The vacuum evolution equations for the general threedimensional case can be found in $[12,13,7]$. The vacuum constraint equations include

$$
\begin{aligned}
& 0=\bar{R}_{i j}-L_{i j}+H K_{i j}-2 K_{i k} K_{j}^{k}-a_{i} a_{j}-a_{i j}, \\
& 0=L_{i}^{i}+K^{i j} K_{i j}+a^{i} a_{i}+a_{i}^{i}, \\
& 0=M^{j}{ }_{j i}-M_{i j}{ }^{j}, \\
& 0=a_{0 i}+H a_{i}+M_{i j}{ }^{j},
\end{aligned}
$$

where $\bar{R}_{i j}$ is the three-dimensional Ricci tensor formed from the three-metric $g_{i j}$. Equations (4a)-(4c) follow from the Gauss-Codazzi-Ricci equations for embedding a foliation into a higher-dimensional space, and Eq. (4d) follows from harmonic time slicing. Additional constraints that must be satisfied at all times are the definitions (2c), (2d), and (2f), and the usual relation between $\Gamma^{k}{ }_{i j}$ and derivatives of $g_{i j}$.

\section{B. Spherical symmetry}

The spherically symmetric three-metric can be written in the general form

$$
{ }^{(3)} d s^{2}=A^{2} d r^{2}+B^{2} r^{2}\left(d \theta^{2}+\sin ^{2} \theta d \phi^{2}\right),
$$

where $(r, \theta, \phi)$ are the usual spherical coordinates. Define

$$
\begin{aligned}
\Gamma_{r T} & \equiv 2 B r \Gamma_{\theta r}^{\theta}=2 B r \Gamma_{\phi r}^{\phi} \\
& =-\frac{2 A^{2}}{B r} \Gamma_{\theta \theta}^{r}=-\frac{2 A^{2}}{B r \sin ^{2} \theta} \Gamma_{\phi \phi}^{r}, \\
a_{T} & \equiv a_{\theta}^{\theta}=a_{\phi}^{\phi}, \\
L_{T} & \equiv L_{\theta}^{\theta}{ }_{\theta}=L_{\phi}^{\phi}, \\
K_{T} & \equiv K_{\theta}^{\theta}=K_{\phi}^{\phi}, \\
M_{r T} & \equiv M_{r}{ }_{\theta}{ }_{\theta}=M_{r}{ }_{\phi}, \\
M_{T r} & \equiv M_{\theta r}^{\theta}=M_{\phi r}^{\phi},
\end{aligned}
$$

where the subscript $T$ denotes "transverse."

The evolution equations can be written in the form

$$
\begin{aligned}
\hat{\partial}_{0} A & =-N A K_{r}^{r}, \\
\hat{\partial}_{0} B r & =-N B r K_{T}, \\
\hat{\partial}_{0} K_{r r} & =N L_{r r}, \\
\hat{\partial}_{0} K_{T} & =N\left(L_{T}+2 K_{T}^{2}\right), \\
\hat{\partial}_{0} N & =-N^{2}\left(K_{r}^{r}+2 K_{T}\right), \\
\hat{\partial}_{0} a_{r} & =N a_{0 r},
\end{aligned}
$$

$$
\begin{aligned}
\hat{\partial}_{0} a_{T}= & N\left[\left(2 M_{T r}-M_{r T}-a_{r} K_{T}\right) \frac{a_{r}}{A^{2}}+\frac{\Gamma_{r T}}{2 A^{2} B r} a_{0 r}\right. \\
& \left.+2 K_{T} a_{T}\right], \\
\hat{\partial}_{0} \Gamma_{r r}^{r}= & -\frac{N}{A^{2}}\left[K_{r r} a_{r}+M_{r r r}\right], \\
\hat{\partial}_{0} \Gamma_{r T}= & -N\left[K_{T} \Gamma_{r T}+2 B r\left(a_{r} K_{T}+M_{r T}\right)\right],
\end{aligned}
$$$$
\hat{\partial}_{0} M_{T r}=N\left[K_{T}\left(2 M_{T r}+M_{r T}+a_{r} K_{T}\right)+\frac{\Gamma_{r T}}{2 B r}\left(\frac{L_{r r}}{A^{2}}-L_{T}\right)\right.
$$$$
\left.+K_{r}^{r}\left(2 M_{T r}-M_{r T}-a_{r} K_{T}\right)\right] \text {, }
$$

$$
\hat{\partial}_{0} a_{r r}=N \frac{\partial}{\partial r} a_{0 r}+N\left[-\Gamma_{r r}^{r} a_{0 r}+a_{r}^{2} K_{r}^{r}+\frac{a_{r} M_{r r r}}{A^{2}}+a_{r} a_{0 r}\right],
$$

$$
\begin{aligned}
\hat{\partial}_{0} a_{0 r}= & \frac{N}{A^{2}} \frac{\partial}{\partial r} a_{r r}+N\left[\frac{M_{r r r}}{A^{2}}\left(2 K_{T}-K_{r}^{r}\right)\right. \\
& +a_{r}\left(\left(a_{r}^{2}-L_{r r}\right) \frac{1}{A^{2}}-2 K_{r}^{r}\left(K_{r}^{r}-3 K_{T}\right)\right) \\
& +\frac{a_{r r}}{A^{2}}\left(3 a_{r}-2 \Gamma_{r r}^{r}+\frac{\Gamma_{r T}}{B r}\right) \\
& \left.+2 M_{r T} K_{r}^{r}+a_{T}\left(4 a_{r}-\frac{\Gamma_{r T}}{B r}\right)\right],
\end{aligned}
$$

$\hat{\partial}_{0} M_{r r r}=N \frac{\partial}{\partial r} L_{r r}+N\left[\left(a_{r}-2 \Gamma_{r r}^{r}\right) L_{r r}+2 K_{r}^{r}\left(K_{r r} a_{r}+M_{r r r}\right)\right]$,

$$
\begin{aligned}
\hat{\partial}_{0} L_{r r}= & \frac{N}{A^{2}} \frac{\partial}{\partial r} M_{r r r}+N\left[L_{r r}\left(4 K_{T}-5 K_{r}^{r}\right)+8 M_{r T} a_{r}\right. \\
& +\frac{M_{r r r}}{A^{2}}\left(3\left(a_{r}-\Gamma_{r r}^{r}\right)+\frac{\Gamma_{r T}}{B r}\right)-\frac{2 M_{T r} \Gamma_{r T}}{B r} \\
& +2 a_{r r}\left(3 K_{T}-K_{r}^{r}\right)+a_{r}^{2}\left(10 K_{T}-K_{r}^{r}\right) \\
& \left.-K_{r r}\left(5 K_{r}^{r 2}-6 K_{r}^{r} K_{T}+2 K_{T}^{2}\right)\right], \\
\hat{\partial}_{0} M_{r T}= & N \frac{\partial}{\partial r} L_{T}+N\left[2 K_{T}\left(a_{r} K_{T}+2 M_{r T}\right)+a_{r} L_{T}\right],
\end{aligned}
$$$$
\hat{\partial}_{0} L_{T}=\frac{N}{A^{2}} \frac{\partial}{\partial r} M_{r T}+N\left[L_{T} K_{r}^{r}+\frac{a_{r}^{2} K_{r}^{r}}{A^{2}}\right.
$$$$
+\left(L_{r r}+a_{r r}\right)\left(K_{r}^{r}-K_{T}\right) \frac{1}{A^{2}}-2 K_{T}^{3}
$$ 


$$
\begin{aligned}
& +2 a_{T}\left(K_{r}^{r}+K_{T}\right)-2 K_{r}^{r 2} K_{T}+K_{r}^{r 3} \\
& +2 K_{r}^{r} K_{T}^{2}+\frac{M_{T r}}{A^{2}}\left(\frac{\Gamma_{r T}}{B r}-4 a_{r}\right) \\
& \left.+\frac{M_{r T}}{A^{2}}\left(\frac{\Gamma_{r T}}{B r}+3 a_{r}-\Gamma_{r r}^{r}\right)\right]
\end{aligned}
$$

The constraints (4) become

$$
\begin{gathered}
\frac{1}{B r}\left[-\frac{\partial}{\partial r} \Gamma_{r T}+\Gamma_{r r}^{r} \Gamma_{r T}\right]-a_{r r}-a_{r}^{2} \\
+K_{r r}\left(2 K_{T}-K_{r}^{r}\right)-L_{r r}=0 \\
\frac{1}{2 A^{2} B r}\left[-\frac{\partial}{\partial r} \Gamma_{r T}+\Gamma_{r r}^{r} \Gamma_{r T}-\frac{\Gamma_{r T}^{2}}{2 B r}\right] \\
+\frac{1}{B^{2} r^{2}}+K_{T} K_{r}^{r}-a_{T}-L_{T}=0 \\
2 L_{T}+\frac{L_{r r}}{A^{2}}+2 K_{T}^{2}+K_{r}^{r 2}+2 a_{T}+\frac{1}{A^{2}}\left(a_{r}^{2}+a_{r r}\right)=0 \\
a_{0 r}+a_{r}\left(2 K_{T}+K_{r}^{r}\right)+\frac{M_{r r r}}{A^{2}}+2 M_{r T}=0
\end{gathered}
$$

The additional constraints $(2 \mathrm{c}),(2 \mathrm{~d}),(2 \mathrm{f})$, and the usual relation between $\Gamma_{i j}^{k}$ and derivatives of $g_{i j}$ take the form

$$
\begin{aligned}
\frac{\partial}{\partial r} K_{r r}-2 \Gamma_{r r}^{r} K_{r r}-M_{r r r} & =0, \\
\frac{\partial}{\partial r} K_{T}-M_{r T} & =0, \\
M_{T r}-\frac{\Gamma_{r T}}{2 B r}\left(K_{r}^{r}-K_{T}\right) & =0, \\
\frac{\partial}{\partial r}(\ln N)-a_{r} & =0, \\
a_{T}-\frac{\Gamma_{r T}}{2 A^{2} B r} a_{r} & =0, \\
\frac{\partial}{\partial r} a_{r}-a_{r r}-\Gamma_{r r}^{r} a_{r} & =0, \\
\frac{\partial}{\partial r} A-A \Gamma_{r r}^{r} & =0, \\
\frac{\partial}{\partial r} B r-\frac{\Gamma_{r T}}{2} & =0 .
\end{aligned}
$$

\section{FREE EVOLUTION OF THE ER SYSTEM}

\section{A. Method}

We solve the spherically symmetric ER evolution equations (7) at every time step using the causal differencing method described in [7]. The constraints are satisfied on the initial time slice but are not solved explicitly during the evolution.

The inner boundary of the numerical domain is a surface that remains within a grid spacing of the apparent horizon, $r=r_{A H}$. Because the apparent horizon is an outgoing null or spacelike surface, the hyperbolic evolution equations require no boundary condition there. The outer boundary is an arbitrary spherical surface far from the black hole at $r=r_{\text {max }}$. At the outer boundary, we use the "extended Robin', condition discussed in [7]. This outer boundary condition does not properly handle wavelike behavior, but in practice it is adequate for the cases shown here.

The lapse function can be freely specified on the initial time slice, and is subsequently determined by the harmonic time slicing condition $\square t=0$. The shift is chosen to satisfy the minimal strain equation [25]. This equation minimizes the average change in the three-metric as one evolves from one time slice to the next, and is used to provide a shift vector that does not produce coordinate singularities. The minimal strain equation requires two boundary conditions, for which we choose

$$
\beta^{r}-\frac{N}{A}=0 \quad \text { at } r=r_{A H},
$$

$$
\frac{\partial}{\partial r}\left(r^{2} \beta^{r}\right)=0 \quad \text { at } r=r_{\max }
$$

The inner boundary condition ensures that at the apparent horizon, the coordinates move outward at the local speed of light, $c=N / A$. This prevents the coordinates from falling into the black hole. The outer boundary condition ensures that the shift falls off like $r^{-2}$, in accordance with the timeindependent Schwarzschild solution written in harmonic slicing [Eqs. (11) below]. We use a feedback technique [7] to keep the horizon near $r=2 M$.

\section{B. Initial data}

Our initial data are chosen on a time slice corresponding to a well-behaved, fully time-independent harmonic foliation of the Schwarzschild geometry (cf. Refs. [26-28]). Such a slice penetrates the event horizon without encountering a coordinate singularity, and extends to the physical singularity at $r=0$. With an appropriate choice of spatial coordinates on the slice, all dynamical variables are time-independent [28] and are given by

$$
\begin{aligned}
A^{2} & =\left(1+\frac{2 M}{r}\right)\left[1+\left(\frac{2 M}{r}\right)^{2}\right], \\
B & =1,
\end{aligned}
$$




$$
\begin{aligned}
N & =\frac{1}{A}, \\
\beta^{r} & =\frac{4 N^{2} M^{2}}{r^{2}}, \\
\Gamma_{r r}^{r} & =-\frac{N^{2} M}{r^{2}}\left[1+\frac{4 M}{r}+\frac{12 M^{2}}{r^{2}}\right],
\end{aligned}
$$$$
\Gamma_{r T}=2,
$$$$
K_{T}=\frac{4 N M^{2}}{r^{3}},
$$$$
K_{r r}=-\frac{4 N M^{2}}{r^{3}}\left[2+\frac{3 M}{r}+\frac{4 M^{2}}{r^{2}}+\frac{4 M^{3}}{r^{3}}\right],
$$$$
M_{r r r}=\frac{4 N^{3} M^{2}}{r^{4}}\left[6+\frac{18 M}{r}+\frac{35 M^{2}}{r^{2}}+\frac{40 M^{3}}{r^{3}}+\frac{56 M^{4}}{r^{4}}\right.
$$$$
\left.+\frac{64 M^{5}}{r^{5}}+\frac{48 M^{6}}{r^{6}}\right]
$$$$
M_{r T}=-\frac{4 N^{3} M^{2}}{r^{4}}\left[3+\frac{5 M}{r}+\frac{8 M^{2}}{r^{2}}+\frac{12 M^{3}}{r^{3}}\right] \text {, }
$$$$
M_{T r}=M_{r T},
$$$$
L_{r r}=-\frac{16 N^{4} M^{4}}{r^{6}}\left[14+\frac{42 M}{r}+\frac{85 M^{2}}{r^{2}}+\frac{120 M^{3}}{r^{3}}\right.
$$$$
\left.+\frac{136 M^{4}}{r^{4}}+\frac{128 M^{5}}{r^{5}}+\frac{80 M^{6}}{r^{6}}\right]
$$$$
L_{T}=\frac{16 N^{4} M^{4}}{r^{6}}\left[1+\frac{M}{r}-\frac{4 M^{3}}{r^{3}}\right] \text {, }
$$$$
a_{r}=\frac{N^{2} M}{r^{2}}\left[1+\frac{4 M}{r}+\frac{12 M^{2}}{r^{2}}\right]
$$$$
a_{T}=\frac{N^{4} M}{r^{3}}\left[1+\frac{4 M}{r}+\frac{12 M^{2}}{r^{2}}\right],
$$$$
a_{r r}=-\frac{M N^{4}}{r^{3}}\left[2+\frac{13 M}{r}+\frac{56 M^{2}}{r^{2}}+\frac{40 M^{3}}{r^{3}}-\frac{48 M^{5}}{r^{5}}\right] \text {, }
$$$$
a_{0 r}=\frac{16 M^{3} N^{5}}{r^{5}}\left[1+\frac{6 M}{r}+\frac{24 M^{2}}{r^{2}}+\frac{24 M^{3}}{r^{3}}+\frac{16 M^{4}}{r^{4}}\right] \text {, }
$$

where $M$ is the mass of the black hole. One can explicitly check the time-independence of this solution by inserting Eqs. (11) into the ER evolution equations (7) and verifying that all time derivatives are zero. Note that Eqs. (11) satisfy the minimal strain shift condition, as does any timeindependent solution of Einstein's equations.

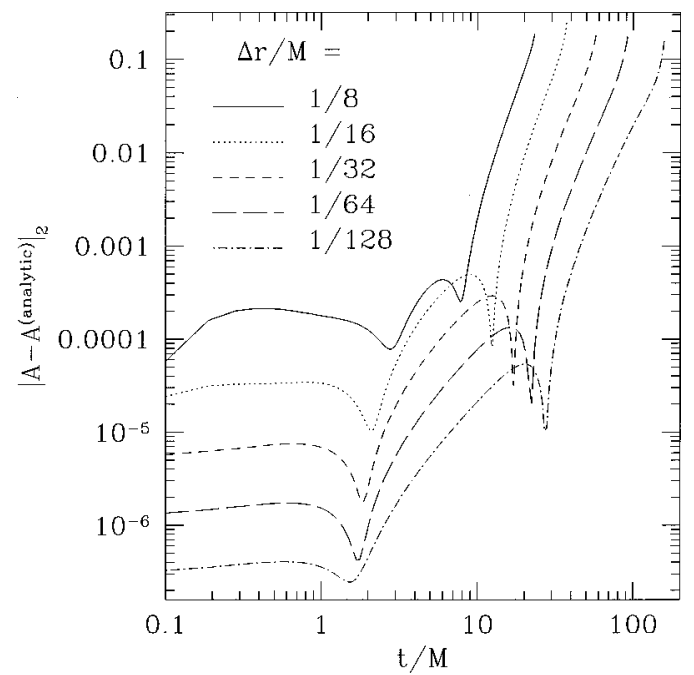

FIG. 1. The $l_{2}$ norm of $A$ minus its analytic solution (11a), shown as a function of time for five grid resolutions. The outer boundary is at $r_{\max }=64 \mathrm{M}$ and the Courant factor $\Delta t / \Delta r$ is $3 / 4$. All five plots terminate when the code crashes.

\section{Results}

Figure 1 shows the error in the metric function $A$ as a function of time. We plot the quantity $\left|A-A^{\text {an }}\right|_{2}$, where $A^{\text {an }}$ is the analytic value of $A$ given by Eq. (11a), and the $l_{2}$ norm of a quantity $q$ is defined by

$$
|q|_{2} \equiv \frac{\sqrt{\sum_{i=1}^{N} q_{i}^{2}}}{N}
$$

The sum is over all grid points that contain valid data (i.e., all grid points outside the horizon). The quantity $\left|A-A^{\mathrm{an}}\right|_{2}$ is shown for several different grid resolutions, each with the same Courant factor $\Delta t / \Delta r$.

At early times, the error in $A$ varies with resolution like $\mathcal{O}(\Delta r)^{2}$, as expected for our second-order convergent numerical method. However, after about $(10-30) M$ the error grows rapidly, approximately like $t^{4}$ at late times. The growth rate is independent of the grid resolution. Eventually, when errors have become sufficiently large, the code crashes, typically because it fails to locate an apparent horizon.

It is common for numerical finite-difference schemes to produce solutions with errors that grow as the truncation error accumulates. However, such growth is typically linear in time, with a slope proportional to $(\Delta t)^{2}$ (for a secondorder scheme), and can be easily defeated by increasing the resolution. In contrast, Fig. 1 shows a more rapid growth rate that increases with time, indicating that we are observing something other than accumulating truncation errors.

In Fig. 2 we plot the error in $A$ as a function of radius for several different times. The error is greatest near the horizon and remains smooth in both space and time as it grows. The fact that our errors are largest near the black hole does not necessarily indicate that the instability is somehow associated with our treatment of the inner boundary; one expects numerical errors to be greater for smaller values of $r$ simply 


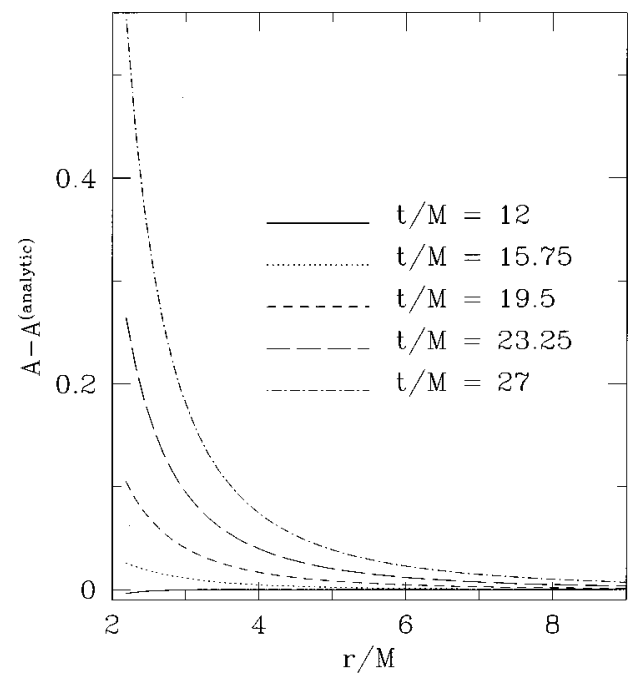

FIG. 2. Error in $A$ as a function of coordinate radius, for the $\Delta r / M=1 / 16$ case shown in Fig. 1 . The function $A-A^{\text {an }}$ is plotted at five times. The error grows rapidly but smoothly until the code crashes.

because most quantities in Eqs. (11) behave like $1 / r^{n}$ with positive $n$.

Other quantities behave much like the error in $A$. In Fig. 3 we plot the error in $L_{r r}$ with respect to the analytic solution (111), and in Fig. 4 we plot the left-hand side of the Hamiltonian constraint (8c). Both quantities are approximately second-order convergent, but at late times they increase rapidly (faster than linearly) in time at a rate independent of the grid resolution.

Figures 1-4 suggest that the instability is not purely numerical. Numerical instabilities typically grow like $e^{n}$, where $n$ is the number of time steps. Consequently, for a numerical instability one expects that reducing the time discretization $\Delta t$ would make the instability grow faster as a function of time, because integrating to a particular value of $t$ requires more steps. However, in Figs. 1-4, $\Delta t$ is decreased with

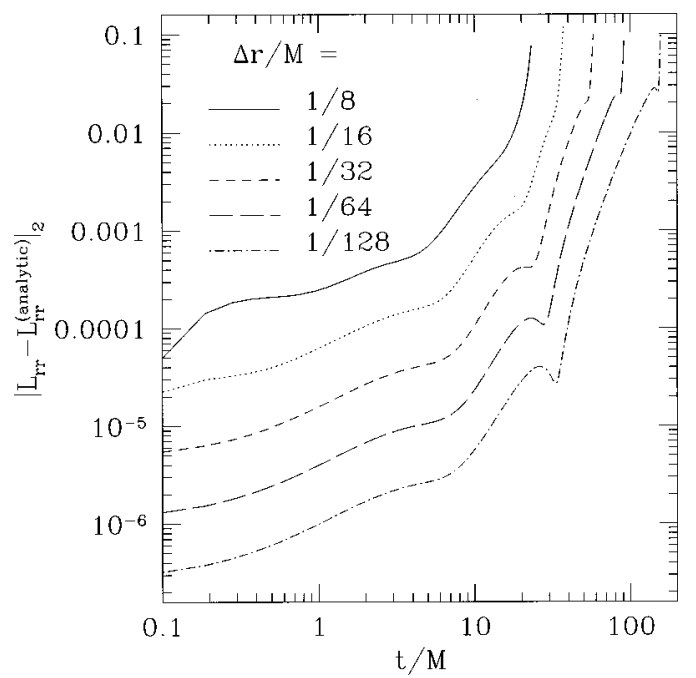

FIG. 3. The $l_{2}$ norm of $L_{r r}$ minus its analytic solution (111) as a function of time, shown for the same cases as plotted in Fig. 1.

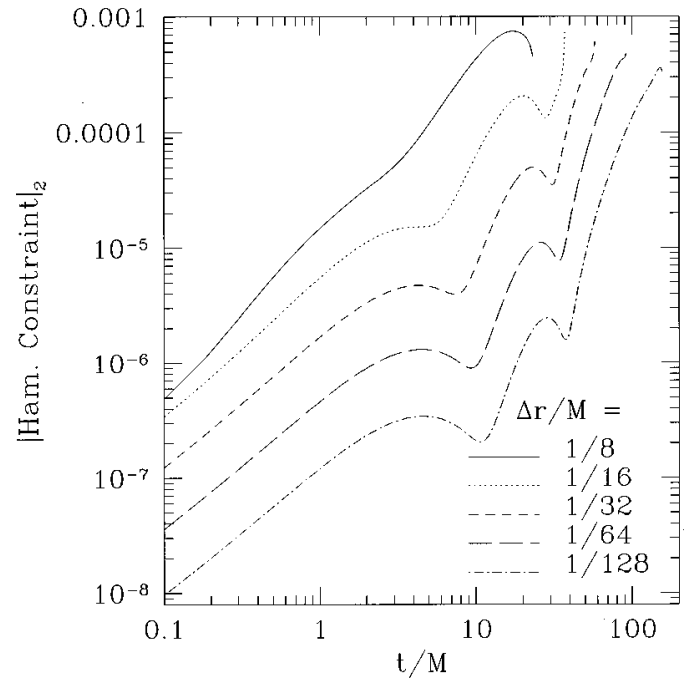

FIG. 4. The $l_{2}$ norm of the Hamiltonian constraint $(8 \mathrm{c})$ versus time, shown for the same five cases as in Fig. 1.

each finer grid resolution, but the growth rate is unaffected. Similarly, at late times we see no change in the growth rate if we vary $\Delta t$ while keeping the grid resolution fixed, as shown in Fig. 5. Instead, for $\Delta t \rightarrow 0$ our errors converge to a limit (this is simply the limit in which numerical truncation error is dominated by $\Delta r$ instead of $\Delta t$ ).

Our results instead suggest that our code suffers from a continuum instability. In this case, the code should remain second-order convergent and the growth rate of errors should depend only on the continuum equations and not on numerical parameters like $\Delta r$ or $\Delta t$. A smaller $\Delta t$ or $\Delta r$ should not intensify the instability, but instead should improve our simulations by virtue of reducing the numerical perturbations that excite the offending mode. Our results are consistent with these expectations.

One possible source of a continuum instability is a rapidly increasing constraint-violating solution of the evolution

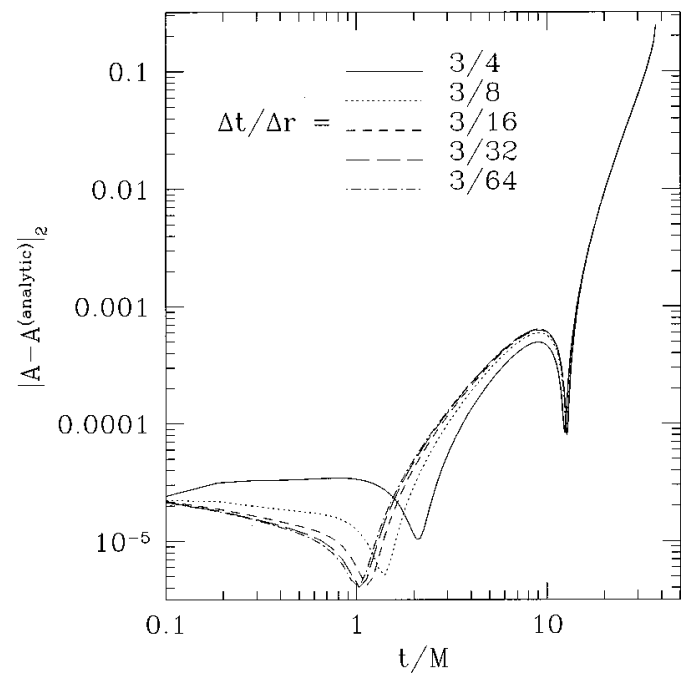

FIG. 5. The $l_{2}$ norm of $A-A^{\text {an }}$ versus time shown for five different values of $\Delta t$, each with $\Delta r / M=1 / 16$. The outer boundary is at $r=64 M$. At late times, the dominant error is independent of $\Delta t$. 
equations that is being excited by numerical perturbations. Another is a gauge mode that is not present in the analytic solution. In the case of a gauge mode, one would expect gauge-invariant quantities to remain relatively unaffected while other quantities blow up. However, at late times, both gauge-dependent quantities like $L_{r r}$ (Fig. 3) and gaugeinvariant quantities like the Hamiltonian constraint (Fig. 4) increase rapidly with time at approximately the same rate.

\section{STABILITY OF INDIVIDUAL EVOLUTION EQUATIONS}

To gain further insight into the nature of the instability, we consider each ER evolution equation separately. For each evolution equation, we treat the ER variable governed by that equation as freely evolving, but we fix the remaining ER variables to the analytic expressions given in Eqs. (11). In this way we can study the stability of each individual evolution equation in the absence of all couplings to other equations. Although this analysis will not shed light on any instabilities that are caused by these couplings, it is likely that if any of the evolution equations are found to be unstable individually, they will remain unstable when coupled to the other equations.

We note that the method of analysis described below can also be used to examine coupled sets of equations as long as the couplings do not arise from derivative terms - this is described in more detail in the Appendix. However, we will see that treating one equation at a time is sufficient for the case discussed here.

Let $y$ represent any of the ER variables that evolve according to Eqs. (7). If all ER variables other than $y$ are considered known functions of $r$, then the evolution equation for $y$ takes the form

$$
\frac{\partial}{\partial t} y-\beta(r) \frac{\partial}{\partial r} y=S(y, r),
$$

where the function $S(y, r)$ contains no derivatives of $y$. If we perturb the quantity $y$ about its time-independent solution by writing $y \rightarrow y+\xi$, then Eq. (13) yields, to first order in $\xi$,

$$
\frac{\partial}{\partial t} \xi-\beta(r) \frac{\partial}{\partial r} \xi=R(r) \xi,
$$

where $R(r)$ does not depend on $\xi$.

For each of the ER evolution equations (7) there is a corresponding perturbation equation of the form (14). Each perturbation equation has a different function $R(r)$ that depends on the right-hand side of the corresponding evolution equation. We will see that the form of $R(r)$ is what determines whether a particular evolution equation is individually stable.

For the simple case in which $\beta(r)$ and $R(r)$ are constants and $\beta>0$, the solution to Eq. (14) on $r \in[2 M, \infty]$ is

$$
\xi(r, t)=\xi_{0}(r+\beta t) e^{R t},
$$

where $\xi_{0}(r)$ is the initial perturbation at $t=0$. The stability is determined by the sign of $R$ : If $R>0$ (assuming that the initial perturbation falls off with radius more slowly than $e^{-r R / \beta}$ ), the perturbation grows exponentially with time; if $R<0$ (assuming that the initial perturbation grows with radius more slowly than $e^{r|R| / \beta}$ ), the perturbation decays.

For the more realistic case of nonconstant $R$ and $\beta$, the solution to Eq. (14) is more complicated than Eq. (15) and is considered in the Appendix. Nevertheless, one can roughly determine whether a given ER evolution equation is individually stable by examining the sign of the function $R(r)$ associated with that evolution equation.

Applying this criterion to the ER evolution equations (7), we find that $R(r)$ is everywhere negative for all but four of these equations, indicating that these equations should be stable to small perturbations. The four remaining equations have positive $R(r)$, suggesting that they might be unstable. If $R(r)_{[y]}$ denotes the function $R(r)$ associated with perturbations of the variable $y$, then the four positive $R(r)_{[y]}$ are

$$
\begin{aligned}
R(r)_{\left[K_{T}\right]} & =4 N K_{T}=\frac{2 z^{3}}{M(1+z)\left(1+z^{2}\right)}, \\
R(r)_{\left[a_{T}\right]} & =2 N K_{T}=\frac{z^{3}}{M(1+z)\left(1+z^{2}\right)}, \\
R(r)_{\left[M_{r T}\right]} & =4 N K_{T}+\frac{\partial}{\partial r} \beta \\
& =\frac{z^{3}\left(2+3 z+4 z^{2}+5 z^{3}\right)}{2 M(1+z)^{2}\left(1+z^{2}\right)^{2}}, \\
R(r)_{\left[L_{r r}\right]} & =N\left(4 K_{T}-5 K_{r}^{r}\right)+2 \frac{\partial}{\partial r} \beta \\
& =\frac{z^{3}\left(20+19 z+18 z^{2}+17 z^{3}\right)}{4 M(1+z)^{2}\left(1+z^{2}\right)^{2}},
\end{aligned}
$$

where $z \equiv 2 M / r$ and the expressions in terms of $z$ have been obtained from the analytic solution (11).

We can test whether perturbations of individual evolution equations are indeed unstable by modifying our code so that a single dynamical variable may be evolved in time while all other variables, including the shift, are held fixed to the analytic solution (11). We find numerically that all evolution equations (7) are individually stable except Eq. (7n), the equation for $L_{r r}$.

Our above analysis predicted that the $L_{r r}$ equation should be individually unstable because it is associated with a positive $R(r)$. However, it also predicted that the $K_{T}, a_{T}$, and $M_{r T}$ equations should be unstable for the same reason. As shown by a more detailed analysis in the Appendix, the $K_{T}$, $a_{T}$, and $M_{r T}$ equations are stable because their corresponding values of $R(r)$ are much smaller in magnitude than the value of $R(r)$ associated with the $L_{r r}$ equation.

The growing mode allowed by the $L_{r r}$ evolution equation (7n) can be described as a continuum instability: it depends only on the equation itself and the equilibrium solution, and 
not on numerics. The only role of numerics is to produce the initial perturbations that excite the unstable mode.

\section{MODIFIED EVOLUTION EQUATIONS}

\section{A. Modifications for stability}

The large positive $R(r)$ associated with perturbations of $L_{r r}$ originates from the term $N L_{r r}\left(4 K_{T}-5 K_{r}^{r}\right)$ that appears on the right-hand side of the $L_{r r}$ evolution equation (7n). This term must be modified if the $L_{r r}$ evolution equation is to be made individually stable. There are several ways to accomplish this.

One possibility is to change variables. If one evolves some quantity $Q L_{r r}$ instead of $L_{r r}$, where $Q$ is some combination of the other ER variables, then perturbations of $Q L_{r r}$ will be governed by Eq. (14) with some new value of $R(r)$. By a careful choice of $Q$ one hopes to obtain a more favorable (more negative) $R(r)$. For example, the evolution equation for the quantity $B^{2} r^{2} L_{r r}$ yields $R(r)=N\left(2 K_{T}\right.$ $\left.-5 K_{r}^{r}\right)+2 \partial \beta / \partial r$, which is still positive but is slightly smaller in magnitude than Eq. (16d). Similarly, the evolution equation for $L_{r}^{r}$ yields $R(r)=N\left(2 K_{T}-3 K_{r}^{r}\right)$. However, there are two reasons why such a procedure is unattractive as the sole method of stabilizing the $L_{r r}$ equation. First, the ER equations are linear in $L_{i j}, M_{i j}^{k}, a_{i j}$, and $a_{0 i}$ (but nonlinear in the other variables), and evolving $Q L_{r r}$ where $Q$ is anything other than the metric functions or the lapse would spoil this linearity. Second, in order to make $R(r)$ nonpositive everywhere by evolving the quantity $B^{n} r^{n} L_{r r} / A^{m}$, it turns out that the required value of $n$ is large enough that $B^{n} r^{n} L_{r r} / A^{m}$ grows with $r$, hampering our ability to impose an accurate outer boundary condition.

Another approach is to use the constraint equations to eliminate the troublesome term that appears on the righthand side of the $L_{r r}$ evolution equation (7n). In order to avoid changing the hyperbolic character of the evolution equations, one must use only constraint equations that are algebraic, that is, those that contain no derivatives. Fortunately, many of the ER constraints are algebraic. For some constraints this is merely a result of spherical symmetry, but several ER constraint equations are algebraic even in the general case of three spatial dimensions plus time. In spherical symmetry, the algebraic constraints are Eqs. (8c), (8d), (8e), (8h), and (8j). An additional algebraic constraint can be formed from Eqs. (8a) and (8b) by eliminating the derivative of $\Gamma_{r T}$, yielding

$$
\begin{gathered}
2 L_{T}-\frac{L_{r r}}{A^{2}}-K_{r}^{r 2}+2 a_{T}-\frac{1}{A^{2}}\left(a_{r}{ }^{2}+a_{r r}\right) \\
-\frac{2}{B^{2} r^{2}}+\frac{\Gamma_{r T}{ }^{2}}{2 A^{2} B^{2} r^{2}}=0 .
\end{gathered}
$$

Because we wish to modify the $L_{r r}$ term on the right-hand side of Eq. (7n) for the case in which all variables except $L_{r r}$ are fixed to the analytic solution, the only relevant algebraic constraints are those that involve $L_{r r}$, namely Eqs. (8c) and (17).
We have found several methods of obtaining an individually stable evolution of $L_{r r}$. These all involve the use of algebraic constraint equations, and some also employ a change of variables. We have had the most success with the following approach: First eliminate $L_{T}$ from Eqs. (8c) and (17) to obtain

$$
\frac{\Gamma_{r T}^{2}-4 A^{2}}{4 B^{2} r^{2}}-a_{r}^{2}-a_{r r}-A^{2}\left(K_{T}^{2}+K_{r}^{r 2}\right)-L_{r r}=0 .
$$

Then write down the evolution equation for the quantity $L_{r}^{r}$, and add $N\left(4 K_{T}-5 K_{r}^{r}\right) / A^{2}$ times Eq. (18) to the right-hand side, yielding

$$
\begin{aligned}
\hat{\partial}_{0} L_{r}^{r}= & \frac{N}{A^{2}} \frac{\partial}{\partial r} M_{r r}^{r}+N\left[2 K_{r}^{r} L_{r}^{r}+8 \frac{M_{r T}}{A^{2}} a_{r}\right. \\
& +M_{r r}^{r}\left(3 a_{r}-\Gamma_{r r}^{r}+\frac{\Gamma_{r T}}{B r}\right)-2 \frac{M_{T r} \Gamma_{r T}}{A^{2} B r} \\
& +\left(5 K_{r}^{r}-4 K_{T}\right) \frac{1}{B^{2} r^{2}}\left(1-\frac{\Gamma_{r T}{ }^{2}}{4 A^{2}}\right) \\
& +\frac{a_{r}^{2}}{A^{2}}\left(6 K_{T}+4 K_{r}^{r}\right)+\frac{a_{r r}}{A^{2}}\left(2 K_{T}+3 K_{r}^{r}\right) \\
& \left.+K_{T}\left(2 K_{r}^{r 2}+3 K_{T} K_{r}^{r}-4 K_{T}^{2}\right)\right]
\end{aligned}
$$

Because we now evolve $L_{r}^{r}$ instead of $L_{r r}$, we also choose to evolve $M_{r r}^{r}$ instead of $M_{r r r}$. This preserves the symmetry between the $L-M$ pairs of evolution equations that make up wave equations. The evolution equation for $M_{r r}^{r}$ is

$$
\hat{\partial}_{0} M_{r r}^{r}=N \frac{\partial}{\partial r} L_{r}^{r}+N\left[a_{r} L_{r}^{r}+2 K_{r}^{r 2} a_{r}+4 K_{r}^{r} M_{r r}^{r}\right] .
$$

Evolving $M_{r r}^{r}$ has an additional advantage: perturbations of $M_{r r}^{r}$ governed by Eq. (19b) have a smaller (more negative) $R(r)$ than perturbations of $M_{r r r}$ governed by Eq. $(7 \mathrm{~m})$, and so perturbations of $M_{r r}^{r}$ should decay more rapidly.

\section{B. Results}

Figures 6-8 show the $l_{2}$ norms of the error in $A$, the error in $L_{r r}$, and the Hamiltonian constraint for simulations in which we solve the modified evolution equations (19) in place of Eqs. (7m) and (7n). The numerical method used in these simulations is identical to the one used to integrate the unmodified evolution equations in Sec. III. We use a larger outer boundary radius, $r_{\max }=128 \mathrm{M}$, to suppress outer boundary difficulties that become important at late times.

For the same grid resolution, our code integrates several orders of magnitude farther in time when using the modified evolution equations than when using the unmodified ones. The large errors that grow on a time scale of $(10-100) M$ in Figs. 1-5 are not present in Figs. 6-8. Instead, numerical errors increase linearly with time (or slower than linearly) for over $10000 M$ until difficulties associated with our treatment 


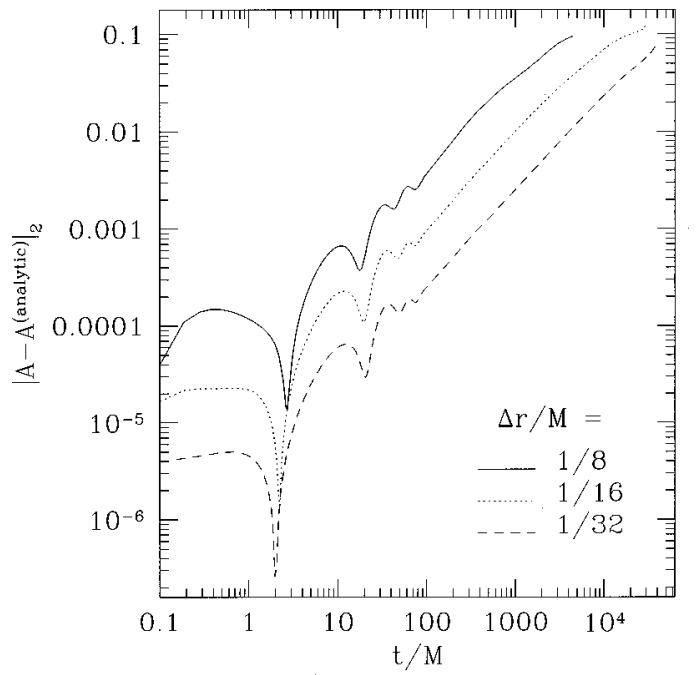

FIG. 6. The $l_{2}$ norm of the error in $A$ versus time, computed for three resolutions using the modified evolution equations. The outer boundary is at $r_{\max }=128 M$ and $\Delta t / \Delta r=3 / 4$. For $t \gtrsim 5 M$ the growth is only linear in time, and the code runs much longer than for the case shown in Fig. 1.

of the outer boundary eventually halt the simulation.

The errors in all dynamical variables except $\mathrm{N}$ and $\mathrm{Br}$ exhibit the same linear growth as seen in Figs. 6 and 7. Errors in $\mathrm{N}$ and $\mathrm{Br}$ are instead dominated by outer-boundary effects that grow rapidly and eventually terminate our code. Figure 9 shows the error in the lapse function $N$ at various times, plotted as a function of radius for several simulations with different outer boundary radii $r_{\max }$ but with the same grid resolution $\Delta r$ and time discretization $\Delta t$. Increasing the outer boundary radius suppresses the rapid growth of outerboundary-related errors at late times and allows for much longer simulations. It should also be possible to improve our results by modifying our outer boundary condition, but the integration times achieved by our code are already beyond

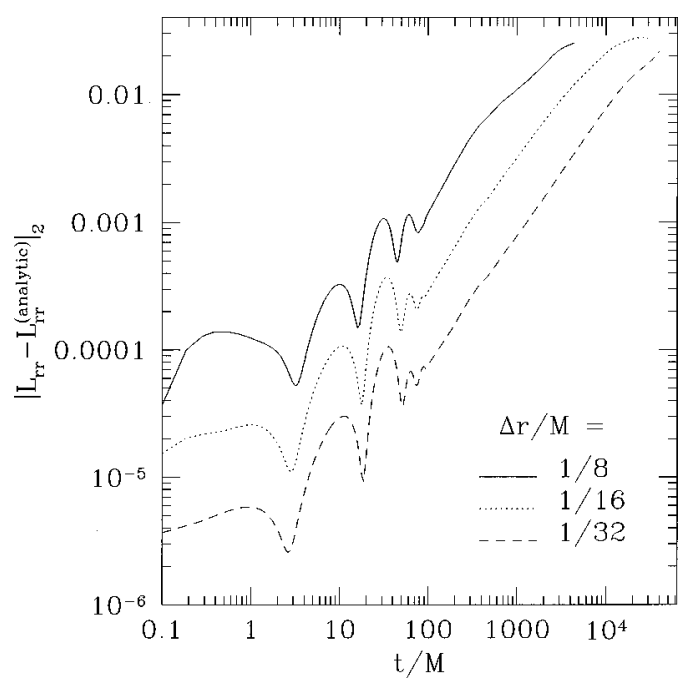

FIG. 7. The $l_{2}$ norm of the error in $L_{r r}$ versus time, shown for the same three cases as in Fig. 6.

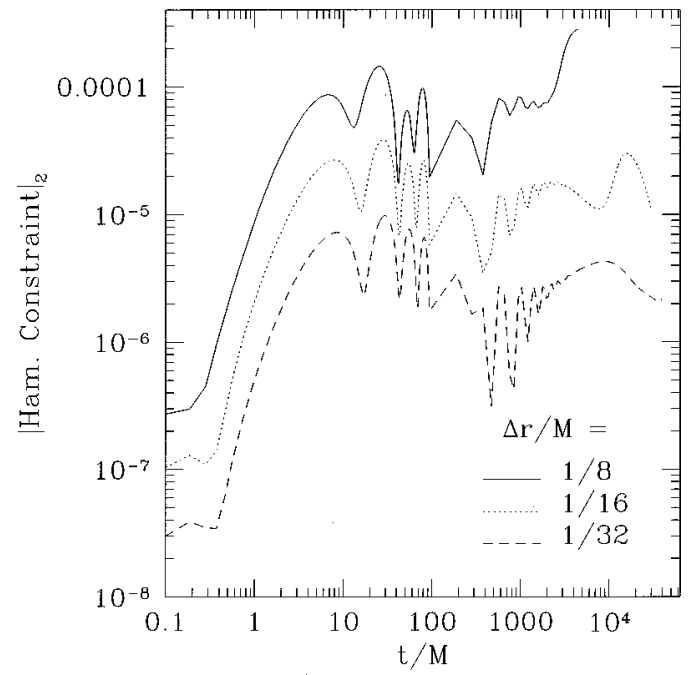

FIG. 8. The $l_{2}$ norm of the Hamiltonian constraint versus time, shown for the same three cases as in Fig. 6. There is no significant growth at late times.

what should be necessary for modeling interesting 3D astrophysical problems such as black-hole binary coalescence.

\section{DISCUSSION}

The success of our free evolution scheme when solving the modified ER equations is strong evidence that the growing continuum mode identified in Sec. IV is responsible for the instability discussed in Sec. III C. The key modification required to suppress the instability was the removal of a term on the right-hand side of the $L_{r r}$ equation, the very term that our analysis in Sec. IV singled out as problematic. Although we have also improved the performance of our code by using $L_{r}^{r}$ and $M_{r r}^{r}$ as dynamical variables instead of $L_{r r}$ and $M_{r r r}$, we have verified that making this change of variables alone, without removing the troublesome term in the $L_{r}^{r}$ equation by

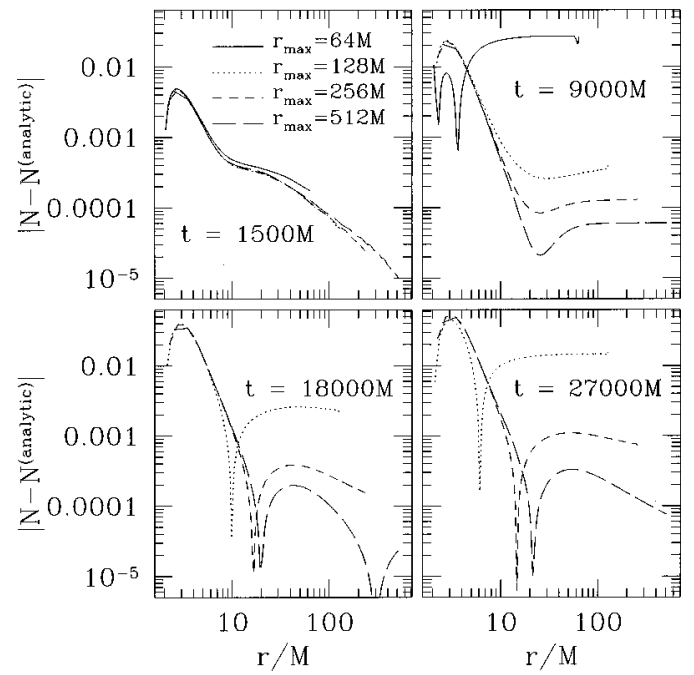

FIG. 9. The absolute value of the error in $N$ as a function of radius, shown at various times for several cases of differing $r_{\max }$. All plots have $\Delta r / M=1 / 32$ and $\Delta t / \Delta r=3 / 4$. The simulation with $r_{\max }=64 M$ crashes at $12000 M$. 
means of constraints, yields results only marginally better than those shown in Sec. III C. Conversely, removing the unstable term and evolving $L_{r r}$ and $M_{r r r}$ instead of $L_{r}^{r}$ and $M_{r r}^{r}$ still allows evolutions to thousands of $M$.

It is no surprise that the detailed behavior of the instability shown in Sec. III C is much more complicated than that predicted by our simple analysis in Sec. IV and in Appendix A. We considered the evolution of a single variable according to a single linear advective equation that possesses only fixed, ingoing (for $\beta>0$ ) characteristic curves. The ER system is actually a coupled system of nonlinear advective and wave equations, and its three families of characteristic curves (along the ingoing and outgoing light cones, and along the normal to the foliation of time slices) depend on the solution. One could do better than our treatment in Sec. IV by linearizing Eqs. (7) about the analytic solution and solving the entire system of coupled linear partial differential equations; however, our approach is far simpler and appears to give the correct qualitative results.

We emphasize that the results presented in Sec. V B were obtained using free evolution, and that no constraints have been enforced. Furthermore, we note that the modifications discussed in Sec. V do not alter the hyperbolic character of the system. A different version of our code evolves Eqs. (7) while enforcing several algebraic constraint equations [specifically, we solve Eq. (8d) for $M_{r T}$, Eqs. (17) and (8c) for $L_{T}$, and Eq. (8e) for $a_{0 r}$ after every time step], and yields evolutions accurate for times on the order of $1000 \mathrm{M}$. While constraint enforcement allows our simulations to remain accurate for far longer times than with free evolution of the unmodified ER equations (7), our partially constrained method eventually succumbs to an instability slightly after $1000 M$. The details of exactly how constraint enforcement suppresses the continuum instability found in Sec. IV are unknown.

We have concentrated on a case in which the analytic solution is manifestly time-independent, namely, when initial data given by Eqs. (11) are evolved using a harmonic time coordinate. However, modifying our evolution equations also dramatically improves our numerical results when initial data are chosen on a minimally modified ingoing EddingtonFinkelstein (MMIEF) [6] time slice, so that subsequent evolution using harmonic time slicing yields a time-dependent result. Using our partially constrained code, we have shown [28] that the evolution of MMIEF initial data using harmonic time slicing relaxes to the solution (11) at late times. The same result holds for free evolution of the modified ER equations.

It is straightforward to extend the analysis in Sec. IV to the three-dimensional ER system. In this case, it is useful to include couplings between tensor components. For example, three-dimensional perturbations of $L_{i j}$, with all other quantities held constant, obey

$$
\frac{\partial}{\partial t} \xi-\beta^{i} \frac{\partial}{\partial x^{i}} \xi=R(x, y, z) \xi
$$

which is similar to Eq. (14) except that here $\xi$ is a column vector containing $\left(\delta L_{x x}, \delta L_{x y}, \ldots, \delta L_{z z}\right)$ and $R(x, y, z)$ is a matrix. For perturbations about the spherically symmetric solution (11), we find that the largest eigenvalue of $R(x, y, z)$ is given by the same expression (16d) as in the spherically symmetric case, indicating that the three-dimensional ER equations should suffer from the same instability as their spherically symmetric counterparts. Applying the same analysis to the $K_{i j}$ evolution equation in the ADM system [using the same analytic solution (11)] results in eigenvalues of $R(x, y, z)$ that are the same size as Eq. (16b) and applying it to the $E_{i j}, H_{k i, j}, K_{i j}$, and $\Gamma_{i j}^{k}$ equations in the EinsteinBianchi system [19] yields eigenvalues of $R(x, y, z)$ that are no larger than $3 / 2$ the size of Eq. (16b); so we expect that the type of continuum instability we find in the ER system should not be present in either of these two other formalisms.

Although our stability analysis makes use of the analytic solution (11), in principle any other solution can be used instead as a background for perturbations. Because the form of the ER evolution equations given by Eqs. (7) assumes harmonic slicing, the only relevant time-independent solution is Eqs. (11). However, for the case of the EinsteinBianchi or ADM system evolved using a different gauge, one might be interested in a different background solution. The features of the background solution that are important for determining stability are the signs and relative magnitudes of components of $K_{i j}$ and derivatives of $\beta^{i}$. We note that these features are approximately the same for the Schwarzschild solution on time-independent MMIEF slices as they are for the Schwarzschild solution on timeindependent harmonic slices, and so one obtains similar stability criteria in both cases.

In the case of the ER equations, we are fortunate to have algebraic constraints that can be used to modify the evolution equations without affecting the hyperbolic character of the system, even in three dimensions. However, not all the ER constraints are algebraic, and it is unclear in the threedimensional case which constraints must be used in order to suppress instabilities. In particular, Eq. (18), which seems necessary for removing the growing mode, is not algebraic in three dimensions. This is because Eq. (18) results from eliminating second derivatives of the metric from Eqs. (8a) and $(8 \mathrm{~b})$; the three-dimensional equivalent is forming a linear combination of components of Eq. (4a) that eliminates all second derivatives of $g_{i j}$ appearing in the Ricci tensor $\bar{R}_{i j}$, and is not possible for a general spacetime.

One might ask why we do not use Eq. (8c) instead of Eq. (18) to obtain a stable evolution equation for $L_{r}^{r}$, since Eq. $(8 \mathrm{c})$ is algebraic in the general three-dimensional case. The answer is that it is possible to use Eq. (8c) to obtain an individually stable evolution equation for $L_{r}^{r}$. However, doing so introduces a term containing $L_{T}$ on the right-hand side of the $L_{r}^{r}$ evolution equation, where no such term existed previously. This term generates a continuum instability in the coupled $L_{r}^{r}$ - $L_{T}$ system (where all variables except $L_{r}^{r}$ and $L_{T}$ are held fixed to the analytic solution).

To better understand why Eq. (8c) alone cannot stabilize the ER equations, consider as fundamental variables not $L_{r r}$ and $L_{T}$, but instead the trace and the trace-free parts of $L_{i j}$, which in spherical symmetry are given by $L_{i}^{i} \equiv L_{r}^{r}+2 L_{T}$ and 
$L^{\mathrm{TF}} \equiv L_{r}^{r}-L_{T}$. If one constructs evolution equations for $L_{i}^{i}$ and $L^{\mathrm{TF}}$, one finds that perturbations of $L^{\mathrm{TF}}$, holding $L_{i}^{i}$ and all other ER variables fixed, obey Eq. (14) with

$$
R(r)=\frac{N\left(10 K_{T}-7 K_{r}^{r}\right)}{3}=\frac{z^{3}\left(48+41 z+34 z^{2}+27 z^{3}\right)}{12 M(1+z)^{2}\left(1+z^{2}\right)^{2}} .
$$

The perturbations grow rapidly with time because $R(r)$ is large and positive. The source of the problem is a large, positive $L^{\mathrm{TF}}$ term on the right-hand side of the $L^{\mathrm{TF}}$ evolution equation. Because Eq. (8c) involves only the trace of $L_{i j}$ and not its trace-free part, this equation cannot be used to eliminate the $L^{\mathrm{TF}}$ term and thus cannot be used to stabilize the system.

If one wishes to use the ER formulation in a 3D free evolution, one must find a way of dealing with the unstable continuum mode afflicting the ER evolution equations. Unfortunately, the above analysis suggests that in $3 \mathrm{D}$, this cannot be done in a simple way using algebraic constraint equations. Accordingly, for 3D simulations it may be more fruitful to pursue other hyperbolic formulations such as the Einstein-Bianchi system, which, according to our analysis, should not suffer from this type of instability.

\section{ACKNOWLEDGMENTS}

We thank Andrew Abrahams, Éanna Flanagan, Pablo Laguna, and James York for helpful discussions. This work was supported by the NSF Binary Black Hole Grand Challenge Grants Nos. NSF PHY 93-18152 and ASC 93-18152 (ARPA supplemented), NSF Grant No. PHY 94-08378 at Cornell, and NSF Grant No. AST 96-18524 and NASA Grant No. NAG 5-3420 at Illinois. Some computations were performed on the Cornell Theory Center SP2 and on the National Center for Supercomputing Applications SGI Origin 2000 .

\section{APPENDIX}

\section{Solution of Eq. (14) on an infinite domain}

Solutions to Eq. (14) propagate along characteristic curves $r=r(t)$ that depend only on the shift vector and are defined by

$$
\frac{d r}{d t}=-\beta(r)
$$

Each spacetime point $(r, t)$ intersects exactly one characteristic curve. If we define $s(r, t)$ to be the radial coordinate at which the characteristic curve passing through $(r, t)$ intersects the initial slice $t=0$, then for $\beta(r)$ given by Eq. (11d) we can integrate Eq. (A1) to find a relation between $s, t$, and $r$ :

$$
\begin{aligned}
\frac{t}{2 M}= & \ln \frac{s}{r}+\frac{1}{3}\left(\frac{r}{2 M}\right)^{3}\left[\left(\frac{s}{r}\right)^{3}-1\right]+\frac{1}{2}\left(\frac{r}{2 M}\right)^{2}\left[\left(\frac{s}{r}\right)^{2}-1\right] \\
& +\frac{r}{2 M}\left[\frac{s}{r}-1\right] .
\end{aligned}
$$

Treating $s$ and $t$ as independent variables, we can write Eq. (14) in the form

$$
\left.\frac{\partial \xi}{\partial t}\right|_{s=\text { const }}=R(r(s, t)) \xi
$$

Each value of $R(r)$ listed in Eqs. (16) can be written in the form

$$
R(r)=\frac{z^{3}\left[a+b z+c z^{2}+(b+c-a) z^{3}\right]}{2 M(1+z)^{2}\left(1+z^{2}\right)^{2}},
$$

where $a, b$, and $c$ are constants and $z \equiv 2 M / r$. Using this expression for $R(r)$ we can integrate Eq. (A3) together with Eq. (A2) to obtain the general solution

$$
\xi(r, t)=\xi_{0}(s)\left(\frac{s}{r}\right)^{a}\left(\frac{1+2 M / r}{1+2 M / s}\right)^{b-a}\left(\frac{1+(2 M / r)^{2}}{1+(2 M / s)^{2}}\right)^{(c-a) / 2}
$$

where $\xi_{0}(r)$ denotes $\xi$ on the initial slice $t=0$.

For a fixed value of $r$ we have $s \gg r$ at late times, and so Eq. (A2) reduces to $t \sim s^{3} / 12 M^{2}$ and Eq. (A5) reduces to

$$
\xi(r, t) \sim \xi_{0}\left(12^{1 / 3} M^{2 / 3} t^{1 / 3}\right)\left(\frac{12 M^{2} t}{r^{3}}\right)^{a / 3},
$$

where time-independent factors have been dropped. If $\xi$ initially falls off like $r^{-m}$, then for a fixed $r$ it will behave like $t^{(a-m) / 3}$ at late times. For $a>m+3$, perturbations will grow superlinearly with time, but for $a \leqslant m+3$ the growth is at most linear (for $a<m$ the perturbation is actually damped), and so it does not represent an instability.

For the $L_{r r}$ equation $(a=10)$ to be individually stable, numerical errors must fall off at least as fast as $r^{-7}$. For the $K_{T}$ equation $(a=4)$ to be stable, the leading-order falloff rate must be no slower than $r^{-1}$. The $M_{r T}$ and $a_{T}$ equations $(a=2)$ will be stable even if numerical errors grow with radius, as long as these errors grow no faster than $r$.

Empirically, we find that the dominant numerical errors in the wavelike variables $\left(L_{r r}, L_{T}, M_{r T}, a_{r r}\right.$, and $\left.a_{0 r}\right)$ fall off like $r^{-1}$ and propagate outward from the strong-field region near the hole. This is what one would expect for modes that behave like gravitational radiation (these modes are not allowed in spherical symmetry but nevertheless can be present in numerical error terms). The dominant errors in other variables also propagate outward from the strong-field region, and fall off either like $r^{-1}$ or $r^{-2}$. These falloff rates explain our observation that the $L_{r r}$ equation is individually unstable but the $K_{T}, M_{r T}$, and $a_{T}$ equations are individually stable.

For background solutions other than Eqs. (11), the forms of $\beta(r)$ and $R(r)$ will be different, and so the details of the solution (A5) will change. For example, if one takes the MMIEF solution as a background [this is not relevant for Eqs. (7) because the MMIEF solution is not preserved under harmonic slicing, but is relevant for other systems of evolution equations to which one might apply this analysis], $R(r)$ typically falls off like $r^{-2}$ instead of $r^{-3}$, and $\beta(r)$ falls off like $r^{-1}$ instead of $r^{-2}$; so the stability criterion becomes 
$a \leqslant m+2$ instead of $a \leqslant m+3$. At the same time, the coefficient $a$ is typically smaller for the MMIEF background; so both the MMIEF background and the background (11) yield similar predictions for stability.

Furthermore, note that our stability criterion can be applied to coupled evolution equations as long as there are no couplings through derivatives. For example, consider the coupled system consisting of all ER variables except $M_{r r r}$, $M_{r T}$, and $a_{0 r}$. If $M_{r r r}, M_{r T}$, and $a_{0 r}$ are held fixed, the perturbation equation for the 13 other variables can be written in the form (14), where in this case $\xi$ is a 13-element column vector and $R(r)$ is a $13 \times 13$ matrix. To determine stability, one examines each eigenmode of the perturbation equation in the manner described above. An example in which this analysis cannot be used without modification is the coupled system consisting of $L_{r r}$ and $M_{r r r}$. In this case, the spatial derivatives of $L_{r r}$ in the $M_{r r r}$ equation $(7 \mathrm{~m})$ and the spatial derivatives of $M_{r r r}$ in the $L_{r r}$ equation (7n) prevent one from writing down a matrix perturbation equation of the form (14). Instead, the perturbation equations possess more than one family of characteristic curves, and so the solution is more complicated.

\section{Solution of Eq. (14) on a finite domain}

In numerical simulations one often does not have a domain that extends to $r=\infty$, but instead one imposes an artificial boundary condition at some finite value of $r$. For simplicity, consider a Dirichlet condition: assume $\xi$ is fixed to some constant value $\xi_{0}$ at the outer boundary $r=r_{0}$. If we let $t_{0}(r)$ be the time it takes for information to propagate from the outer boundary $r_{0}$ to some radius $r<r_{0}$, then for $(t, r)$ such that $t>t_{0}(r)$, the solution of Eq. (14) is timeindependent, and for $(t, r)$ such that $t<t_{0}(r)$, the solution is the same as for the case considered in above, in the first subsection of this appendix.

For $\beta(r)$ given by Eq. (11d), the time it takes for information to propagate inward from radius $s$ to radius $r<s$ is given by Eq. (A2). In this case, for $R(r)$ given by Eq. (A4) the time-independent solution is

$$
\xi(r)=\xi_{0}\left(\frac{r_{0}}{r}\right)^{a}\left(\frac{1+2 M / r}{1+2 M / r_{0}}\right)^{b-a}\left(\frac{1+(2 M / r)^{2}}{1+\left(2 M / r_{0}\right)^{2}}\right)^{(c-a) / 2} .
$$

For $r_{0} \gg r$, the time-independent solution behaves roughly like $r^{-a}$.

One consequence of the above analysis is that if one uses a Dirichlet outer boundary condition and an unstable mode of this type is present (that is, if numerical perturbations fall off more slowly than $r^{-a}$ ), then the instability will become more severe if the outer boundary location is moved to a larger radius. This is because the unstable mode has more time to grow before the time-independent state is reached. We have verified this numerically for the simple case of the $L_{r r}$ evolution equation solved with all other variables held constant.
[1] E. Seidel and W.-M. Suen, Phys. Rev. Lett. 69, 1845 (1992).

[2] P. Anninos et al., Phys. Rev. D 51, 5562 (1995).

[3] M. A. Scheel, S. L. Shapiro, and S. A. Teukolsky, Phys. Rev. D 51, 4208 (1995).

[4] M. A. Scheel, S. L. Shapiro, and S. A. Teukolsky, Phys. Rev. D 51, 4236 (1995).

[5] C. Bona, J. Massó, and J. Stela, Phys. Rev. D 51, 1639 (1995).

[6] R. L. Marsa and M. W. Choptuik, Phys. Rev. D 54, 4929 (1996).

[7] M. A. Scheel et al., Phys. Rev. D 56, 6320 (1997).

[8] G. B. Cook et al., Phys. Rev. Lett. 80, 2512 (1998).

[9] R. Gomez et al., Phys. Rev. Lett. 80, 3915 (1998).

[10] R. Arnowitt, S. Deser, and C. W. Misner, in Gravitation: An Introduction to Current Research, edited by L. Witten (Wiley, New York, 1962), pp. 227-265.

[11] S. Frittelli and O. Reula, Commun. Math. Phys. 166, 221 (1994).

[12] Y. Choquet-Bruhat and J. W. York, Jr., C. R. Acad. Sci., Ser. I: Math. A321, 1089 (1995).

[13] A. Abrahams, A. Anderson, Y. Choquet-Bruhat, and J. W. York, Jr., Phys. Rev. Lett. 75, 3377 (1995).
[14] C. Bona, J. Massó, E. Seidel, and J. Stela, Phys. Rev. Lett. 75, 600 (1995)

[15] M. H. P. M. van Putten and D. M. Eardley, Phys. Rev. D 53, 3056 (1996).

[16] S. Frittelli and O. A. Reula, Phys. Rev. Lett. 76, 4667 (1996).

[17] H. Friedrich, Class. Quantum Grav. 13, 1451 (1996).

[18] F. B. Estabrook, R. S. Robinson, and H. D. Wahlquist, Class. Quantum Grav. 14, 1237 (1997).

[19] A. Anderson, Y. Choquet-Bruhat, and J. W. York, Jr., Topol. Methods Nonlinear Anal. (to be published), gr-qc/9710041.

[20] M. Alcubierre, Phys. Rev. D 55, 5981 (1997).

[21] M. Alcubierre and J. Massó, Phys. Rev. D 157, 4511 (1998).

[22] S. Frittelli, Phys. Rev. D 55, 5992 (1997).

[23] C. Gundlach and J. Pullin, Class. Quantum Grav. 14, 991 (1997).

[24] L. M. Burko and A. Ori, Phys. Rev. D 56, 7820 (1997).

[25] L. L. Smarr and J. W. York, Jr., Phys. Rev. D 17, 2529 (1978).

[26] C. Bona and J. Massó, Phys. Rev. D 38, 2419 (1988).

[27] C. Bona and J. Massó, Phys. Rev. D 40, 1022 (1989).

[28] G. B. Cook and M. A. Scheel, Phys. Rev. D 56, 4775 (1997). 